\title{
Primary Cardiac Tumours: Our Experience
}

\author{
Sharma J, Sherchan S, Pradhan S, Rajbanshi B, Koirala R, Timala R, Pokharel J, Uprety MR, Koirala B
}

Department of Cardiac surgery and Anaesthesiology

Shahid Gangalal National Heart Centre, Kathmandu, Nepal.

\section{Address for Correspondence:}

Jyotindra Sharma

Department of Cardiovascular Surgery

Shahid Gangalal National Heart Centre

Post Box No. 11360

Kathmandu, Nepal.

e-mail: jyotindras@hotmail.com

\begin{abstract}
Introduction

Primary cardiac tumours are rare with autopsy incidence of less than 0.1 percent. We present our experience on surgical treatment of such tumours.

\section{Methods}

Since 2001, fifteen patients underwent surgical intervention for primary cardiac tumour at our centre. Mean age was $46.5 \pm 17.5$ years (range 20 to 73 years). There were eight female patients. Thirteen patients had atrial myxoma and the remaining two had primary malignant tumours. Surgical excision of the tumour was done under cardiopulmonary bypass and cardioplegic cardiac arrest.

Results

Complete excision was possible in all benign cardiac tumours where as excision was possible in only one of the two malignant tumours. One patient with suspected angiosarcoma had biopsy taken from the heart. There was no operative mortality. All the patients with atrial myxoma were symptom free and free of recurrence on follow-up echocardiography. The patient with suspected angiosarcoma (histopathology was reported as hemangioma only) presented with disseminated disease and metastasis to thoracic spine with paraplegia three months postoperatively. The other patient with rhabdomyosarcoma who had complete excision of tumour followed by repair of the defect is currently on adjuvant chemotherapy.
\end{abstract}

\section{Conclusion}

Surgical excision of primary benign cardiac tumours is possible with excellent surgical outcome where as outcome is guarded in primary malignant cardiac tumours.

\section{Key Words}

Cardiac tumours, Myxoma, Rhabdomyosarcoma, Angiosarcoma 


\section{Introduction}

Primary cardiac tumours are rare with an autopsy incidence ranging from $0.001 \%$ to $0.03 \%{ }^{1}$. Modern cardiac imaging transformed primary cardiac tumours from a condition rarely diagnosed before autopsy to a curable form of disease 2. About $2 / 3^{\text {rd }}$ of all primary cardiac tumours are benign and among them atrial myxomas are the most common ${ }^{3}$.Primary cardiac tumours present with a variety of symptoms like arrhythmia, embolization, dyspnoea or sudden death determined by intracardiac location of the tumour ${ }^{4}$. Transthoracic echocardiography is usually diagnostic ${ }^{5}$. Sometimes Transesophageal echocardiography, Magnetic Resonance Imaging and Computed Tomography studies are needed for the diagnosis ${ }^{6}$. Treatment modality depends on the type of the tumour. Surgical excision is indicated in all myxomas. The prognosis of primary malignant tumours is very poor even in cases where surgical excision is possible $e^{7,8,9}$. The goal of surgical excision in sarcomas should be complete resection with preservation and restoration of normal cardiac function since median survival of patients with complete resection of tumour is higher than those with incomplete resection ${ }^{7}$.Adjuvant radiotherapy and chemotherapy has been reported to prolong survival and help in palliation.

We present our surgical experience of 15 cases of primary cardiac tumours since 2001.

\section{Patients and Methods}

Since 2001, fifteen patients underwent surgical intervention for primary cardiac tumour at our centre. Demographics, clinical presentation, diagnostic data, operative data, post operative and follow up data were collected from the patient's medical records and reviewed.

Mean age of the patients was $46.5 \pm 17.5$ years (range 20 to 73 years). There were 8 (53.3\%) female patients. There were 13 patients with atrial myxoma of which two were right sided tumours. Two patients had primary malignant tumour, a rhabdomyosarcoma and a suspected angiosarcoma.

\section{Preoperative evaluation}

The preoperative symptoms were dypnoea (60\%), palpitation (53.3\%), embolism (6.7\%), recurrent pericardial effusion (6.7\%), and incidental finding on routine examination (13.3\%). Majority of the patients with dyspnoea were in New York Heart Association (NYHA) class II. None of the patients with myxoma had family history of myxoma or fell into the group of Carney's complex.

Routine hematological, biochemistry, chest X-ray and electrocardiographic studies were performed in all patients for preoperative evaluation. Diagnosis was made with Transthoracic Echocardiography (TTE) and Transesophageal Echocardiography (TEE) when needed. Average size of myxoma on Echocardiography was $5.2 \pm 2.1 \times 3.1 \pm 1.4$ $\mathrm{cm}$. Ultrasound of the abdomen was done in patients with right sided tumours. Magnetic Resonance Imaging (MRI) was done on patients with malignant tumours. Coronary angiography (CAG) was performed in 4 patients. One patient had insignificant coronary lesion, another one had $90 \%$ stenosis of left circumflex artery and the remaining two patients had normal coronaries.

\section{Operative Procedure}

All the fifteen patients underwent surgical intervention under urgent basis soon after the diagnosis was made.

\section{Myxomas}

All the patients had complete excision of the tumour through median sternotomy under cardiopulmonary bypass and cold blood cardioplegic cardiac arrest. The tumours were excised along with a wide margin of tissue surrounding the attachment of the tumour stalk to avoid recurrence. All the left sided myxomas were excised using biatrial approach while right sided ones were approached via right atrium only. The resulting defect in the interatrial septum (IAS) was repaired with pericardial patch in 9 $(69.2 \%)$ patients and the remaining patients had direct closure.

Five patients underwent additional procedures for associated problems. Four patients had mitral valve repair for associated mitral regurgitation, one patient had tricuspid valve repair along with mitral and one patient underwent coronary artery bypass grafting.

The diagnosis of myxoma was histopathologically confirmed in all the cases.

\section{Malignant Tumours}

Excision of the tumour was possible in only one of the two malignant tumours.

The patient, in whom surgical excision was possible, was a 24 years old female. The intraoperative findings were intratrial fleshy and fungating highly vascular tumour occupying almost whole of left atrium, arising from the left atrial roof and infiltrating the IAS, right atrial free wall and medial aspect of the superior Venacava (SVC). Pulmonary veins were free from tumour extension. She underwent surgical excision of tumour and mitral valve repair through 
median sternotomy under cardiopulmonary bypass and cardioplegic cardiac arrest. The tumour along with roof of left atrium, $2 / 3^{\text {rd }}$ of anterior and medial wall of SVC, whole of IAS and adjacent medial free wall of right atrium were excised. Left atrium reconstruction along with closure of IAS defect was done with pericardial patch. Right atrioplasty and reconstruction of SVC with right atrial appendage was done. The histopathological diagnosis of the tumour was rhabdomyosarcoma.

The other patient, a 26 years old male who presented with recurrent pericardial effusion had operative finding of 2.5 litres of hemorrhagic fluid in the pericardium and multiple highly vascular nodules all over the heart surface. Excision was not possible and biopsy of the tumour with creation of bilateral pericardio-pleural window was done. Histopathology was reported to be hemangioma only but he presented with disseminated disease only 3 months later.

\section{Results:}

Myxomas: There was no operative or in-hospital mortality. One patient developed atrial arrhythmia in intensive care unit (ICU) post operatively and was managed with overdrive atrial pacing. Two patients needed ianotropic support for 3 days. Average ICU stay was $1.7 \pm 0.7$ days. The patients were discharged on an average $5^{\text {th }}$ postoperative day (range 3-9 days).

Follow up data was available from 3 months to 5 years. All the patients were symptom free in NYHA class I and free of recurrence on follow up Echocardiography. Out of the 5 patients who had mitral valve repair, 3 patients do not have any mitral regurgitation (MR) while one has mild and the other moderate MR. No late mortality has been reported.

\section{Malignant Tumours}

There was no operative mortality or complications. The patient with rhabdomyosarcoma is 4 months post surgery. She is in NYHA class I and does not have recurrence of tumour at 3 month's follow up Echocardiography. She is presently on adjuvant chemotherapy and radiotherapy.

The other patient with suspected angiosarcoma (histopathology was reported as hemangioma only) presented with disseminated disease and metastasis to the thoracic spine with paraplegia three months postoperatively. He was in NYHA class III - IV.

We will need a longer follow up to see the late survival.

\section{Discussion}

Cardiac tumours of the heart are rare ${ }^{1-7}$. With improved cardiac imaging and the development of cardiopulmonary bypass to enable intracardiac surgery, cardiac tumours have changed from a postmortem curiosity to a readily diagnosed and frequently treatable form of disease ${ }^{7}$. Myxomas are the commonest benign cardiac tumours comprising approximately half of all benign tumours. Thirteen patients $(86.7 \%)$ in this series were benign tumours and all of those were atrial myxomas which is a higher incidence compared to other studies.

Most of our patients presented with Dyspnoea (60\%) and palpitation (53.3\%). Most of the other series also have reported these symptoms to be the main presenting complains. However, the incidence of embolic events and neurologic symptoms (6.7\%) were much lower than other series. The incidence of asymptomatic patients $(13.3 \%)$ is similar to other reports ${ }^{6}$.

Among the malignant tumours, about $3 / 4^{\text {th }}$ are sarcomas, the commonest being angiosarcoma followed by rhabdomyosarcoma ${ }^{3,6}$. The two patients in our series with malignant tumours were also suspected angiosarcoma( though the histopathology was reported as hemangioma only, looking at the operative finding and the fact that he developed metastasis to thoracic spine 3 months post op, we think it is angiosarcoma) and rhabdomyosarcoma. They presented with dyspnoea and recurrent pericardial effusion. Both of them had palpitation. Others have reported similar findings.

Echocardiography is most of the time diagnostic. TTE can determine the size, shape, location, attachment, number and mobility of a tumour. TEE and MRI is sometimes needed for the confirmation of the diagnosis. Ultrasound of the abdomen should be done in right sided tumours to exclude primary tumours in the abdomen.

Surgical excision is the only effective therapeutic option for patients with cardiac myxoma ${ }^{10}$.We use cardiopulmonary bypass and cardioplegic cardiac arrest through a median sternotomy for complete excision of the tumour. Some surgeons have started using minimal access surgery for the excision of myxomas with comparable results to other series ${ }^{2,11}$. We use biatrial approach for the left sided myxomas and right atrial approach for right sided tumours. We excise a part of the IAS or part of the atrial wall where the tumour is attached to avoid recurrence and repair the defect either with a pericardial patch $(69.2 \%)$ or direct closure depending on the size of the defect. We feel that with biatrial approach, we can have adequate exposure and excise the tumour completely to minimize the recurrence. Some surgeons prefer only left atrial approach for the excision of left sided myxomas. So the optimal operative 
approach to the atrial myxoma lacks uniformity.

Recurrence of cardiac myxoma is reported at around 3\% to $5 \%$ in some series ${ }^{10,12}$. Recurrence has been related to incomplete excision, multi-focality and shedding of tumour fragments ${ }^{10}$. We do not have any recurrence of myxoma in our patients. This may be because our follow up is of shorter duration, none of the patients fall into Carney complex and we excise a cuff of normal atrial tissue at the stalk of the tumour.

The prognosis of patients with primary malignant cardiac tumours is poor with death occurring within 6 months postoperatively on average ${ }^{1}$. Malignant cardiac tumours tend to be diagnosed after they have become extensive and the patients already have a grave prognosis ${ }^{13}$. Many of those tumours may be curable if detected at an earlier stage ${ }^{13}$.The extensive involvement precluding resection or anatomic location hindering access for complete resection and reconstruction have made surgical results unsatisfactory regardless of the techniques used ${ }^{9,8}$. Moreover, it is not known whether adjuvant chemotherapy and radiotherapy may be beneficial in patients in whom "curative" surgery has been performed ${ }^{9,14}$. However, surgical resection of tumour should be tried for the definitive histological diagnosis, to relieve the intracardiac obstruction and allow time for effective adjuvant chemotherapy and radiotherapy. There are reports of increased survival in patients in whom surgical excision of tumour is possible compared to those where resection is not performed. In the two patients we had, we were able to excise the tumour only in one patient and she is currently on adjuvant chemotherapy and radiotherapy. The other patient, in whom the excision was not possible, has multiple metastases with a very bad prognosis. A longer follow up will tell us how the tumour will behave in future in those patients.

\section{Conclusion}

Surgical excision of primary benign cardiac tumours is possible with excellent surgical outcome and is the procedure of choice. Although the outcome is guarded in primary malignant cardiac tumours, surgical excision should be tried whenever possible. The therapeutic approach should include surgery, chemotherapy and radiotherapy in the hope of achieving cure or long term palliation.

\section{References:}

1. Centofanti P, Rosa EDI, Deorsola L, et al. Primary cardiac tumors: early and late results of surgical treatment in 91 patients. Ann Thorac Surg 1999;68:1236-41.

2. Bossert T, Gummert JF, Battellini R, et al. Surgical experience with 77 primary cardiac tumors. Interact CardioVasc Thorac Surg 2005;4:311-315.

3. Kirklin JW, Barratt-Boyes BG. Cardiac surgery, $3^{\text {rd }}$ ed. Churchill Livingstone, 2003:1679-1699.

4. Reynen K. Cardiac myxomas. N Engl J Med 1995;333:1610-7.

5. Fyke FE $3^{\text {rd }}$, Seqard JB, Edwards WD, et al. Primary cardiac tumors: experience with 30 consecutive patients since the introduction of two dimensional echocardiography. J Am Coll Cardiol 1985; Jun;5(6):1465-73.

6. Butany J, Nair V, Naseemuddin A, et al. Cardiac tumours: diagnosis and management. Lancet Oncol 2005;6:219-28.

7. Sabiston D, Spencer F. Surgery of the Chest, $7^{\text {th }}$ ed. Elsevier Saunders, 2005:1741-1748.

8. Filho JDF, Lucchese FA, Leaes $P$, et al. Primary Cardiac Angiosarcoma. A therapeutical dilemma. Arq Bras Cardiol 2002;78(6):589-91.

9. Bakaeen FG, Reardon MJ, Coselli JS, et al. Surgical outcome in 85 patients with primary cardiac tumors. The American Journal of Surgery 2003;186:641-647.

10. Sultan FAT, Syed A, Kazmi K, et al. Cardiac myxomas - clinical spectrum and outcome. JCPSP 2006;16(8):501-503.

11. Ravikumar E, Pawar N, Gnanamuthu R, et al. Minimal access approach for surgical management of cardiac tumors. Ann Thorac Surg 2000;70:1077-9.

12. Sellke FW, Lemmer JH Jr, Vandenberg BF, et al. Surgical treatment of cardiac myxomas: long-term results. Ann Thorac Surg 1990;50:557-61.

13. Perchinsky MJ, Lichtenstein SV, Tyers GFO. Primary cardiac tumors: forty years' experience with 71 patients. Cancer 1997;79:1809-1815.

14. Agrawal V, Agrawal SK, Srivastava Ak, et al. Primary cardiac tumours: Surgical experience and follow-up. Indian Heart $J$ 2003;55:632-636. 